\title{
Föhrenverjüngung: Plastizität und Akklimatisation in einem trockeneren Klima
}

\author{
Barbara Moser \\ Christoph Bachofen
}

Thomas Wohlgemuth
Eidgenössische Forschungsanstalt für Wald, Schnee und Landschaft $(\mathrm{CH})$ * Eidgenössische Forschungsanstalt für Wald, Schnee und Landschaft und

Departement Umweltsystemwissenschaften, ETH Zürich (CH)

Eidgenössische Forschungsanstalt für Wald, Schnee und Landschaft $(\mathrm{CH})$

\section{Föhrenverjüngung: Plastizität und Akklimatisation in einem trockeneren Klima}

\begin{abstract}
Zunehmende Sommertrockenheit könnte die natürliche Verjüngung von Waldföhrenbeständen in den Tieflagen des Rhonetals gefährden. Gartenexperimente am Fuss der Felsensteppe haben gezeigt, dass Keimung und Etablierung der Waldföhre vor allem durch ausbleibende Frühjahrsniederschläge und in geringerem Masse durch Sommertrockenheit oder steigende Temperaturen limitiert sind. Die Keimlinge reagierten rasch auf Trockenperioden, indem sie das Wurzelwachstum auf Kosten des Sprosswachstums steigerten. Im zweiten Jahr waren die Sämlinge bereits gegen Trockenheit gewappnet, sodass eine ausgedehnte Frühjahrs- und Sommertrockenheit, wie sie in Sion in den letzten 154 Jahren zwölfmal vorkam, nur zu minimer Mortalität führte. Erst eine lange Sommerdürre - kein Wasser von Juni bis September - liess 14.7\% der Waldföhrensämlinge absterben. Erstaunlicherweise konnten sich die überlebenden Individuen jedoch selbst an solch extreme Trockenheit akklimatisieren. Nach einer wiederholten Sommerdürre im dritten Jahr sank die Mortalität auf unter $5 \%$. Generell reagierten die Waldföhrensämlinge sehr plastisch, d.h., sie passten ihren Wuchs den gegebenen Umweltbedingungen an. Eine genetische Anpassung war ebenfalls festzustellen: Waldföhren aus Gebieten mit ausgeprägter Sommertrockenheit, darunter jene aus den Tieflagen des Rhonetals, produzierten in allen Behandlungsvarianten mehr Biomasse als solche aus feuchteren Regionen. Die Schwarzföhren reagierten ähnlich wie die Waldföhren, wuchsen jedoch schneller und überlebten die erste Sommerdürre deutlich zahlreicher. Diese Resultate zeigen, dass die Waldföhren der Tieflagen des Rhonetals zu den trockenresistentesten Herkünften Europas gehören. Dank ihrer Plastizität und ihrer Fähigkeit, sich in kurzer Zeit an Trockenheit anzupassen, dürfte natürliche Verjüngung in den Tieflagen des Rhonetals auch in Zukunft stattfinden, wenn auch vielleicht seltener als heute.
\end{abstract}

Keywords: drought, plasticity, acclimation, tree seedlings, Pinus sylvestris, Pinus nigra doi: $10.3188 /$ szf.2018.0269

*Zürcherstrasse 111, CH-8903 Birmensdorf, E-Mail barbara.moser@wsl.ch

$\mathrm{I}$ n den letzten 20 Jahren haben ausgeprägte Trockenperioden in den Tieflagen des Wallis wiederholt zu erhöhten Absterberaten bei adulten Waldföhren (Pinus sylvestris) geführt, so zum Beispiel nach den trockenen Jahren 1998, 2003 und zuletzt 2015/2016 (Rigling et al 2018, dieses Heft). Dass auch die Verjüngung unter den Trockenphasen litt, zeigten flächendeckende Aufnahmen nach dem Waldbrand in Leuk 2003: Auf die extreme Trockenheit von 2003 folgten zwei weitere Jahre mit negativer klimatischer Wasserbilanz (Rigling et al 2018, dieses Heft), die dazu führten, dass sich die Waldföhre nach dem Brand vorerst nur zögerlich etablierte (Moser et al 2010). Diese Beobachtung warf die Frage auf, ob die Verjüngung der Waldföhre in den Tieflagen des Rhonetals in einem zukünftig wärmeren und damit trockeneren Klima gefährdet ist. In Spanien, wo die Waldföhre ihre südlichste Verbreitung erreicht, wurde dieser Frage schon länger nachgegangen. Dort hat sich gezeigt, dass die Überlebenschance von Waldföhrenkeimlingen steigt, wenn sie früh im Jahr keimen und damit genügend Zeit haben, ihr Wurzelsystem zu entwickeln, bevor die Sommertrockenheit einsetzt (Castro 2006). Die in diesen Studien untersuchten Waldföhrenpopulationen sind zwar die südlichsten ihrer Art, doch wachsen sie ausschliesslich in Hochlagen oberhalb 1000 m ü. M., wo bis Ende Mai ausgiebig Niederschläge fallen (Moser et al 2017). Demgegenüber sind in den Zentralalpen ausgedehnte Trockenperioden in den Frühjahrsmonaten keine Seltenheit. Deshalb muss genau abgeklärt werden, ob einheimische Baumarten mediterraner Herkunft unsere Wälder trockenresistenter machen können. Es ist bekannt, dass gewisse Wald- 
föhrenherkünfte genetisch besser an Trockenheit angepasst sind als andere (lokale Anpassung; Gugerli et al 2016). Langlebige Organismen wie Bäume können jedoch auch eine hohe phänotypische Plastizität aufweisen, d.h., genetisch identische Individuen (z.B. Stecklinge) haben je nach Umweltbedingungen ein anderes Erscheinungsbild, das ihr Wachstum und ihre Überlebenschance unter den herrschenden Umweltbedingungen verbessert. Schnelles Sprosswachstum zum Beispiel erhöht die Konkurrenzfähigkeit eines Sämlings, während ein ausgedehntes Wurzelwerk bei Trockenheit einen besseren Zugang zu Wasser und Nährstoffen gewährleistet. Ob das Erscheinungsbild (Phänotyp) von Individuen einer Population genetisch festgelegt ist oder sich entsprechend den Umweltbedingungen verändern kann, hat einen entscheidenden Einfluss darauf, wie sich eine Population an ändernde Umweltbedingungen anpassen kann. Populationen mit einer hohen phänotypischen Plastizität können auf sich ändernde Umweltbedingungen rasch reagieren (Nicotra et al 2010), während genetische Anpassung langsam, über

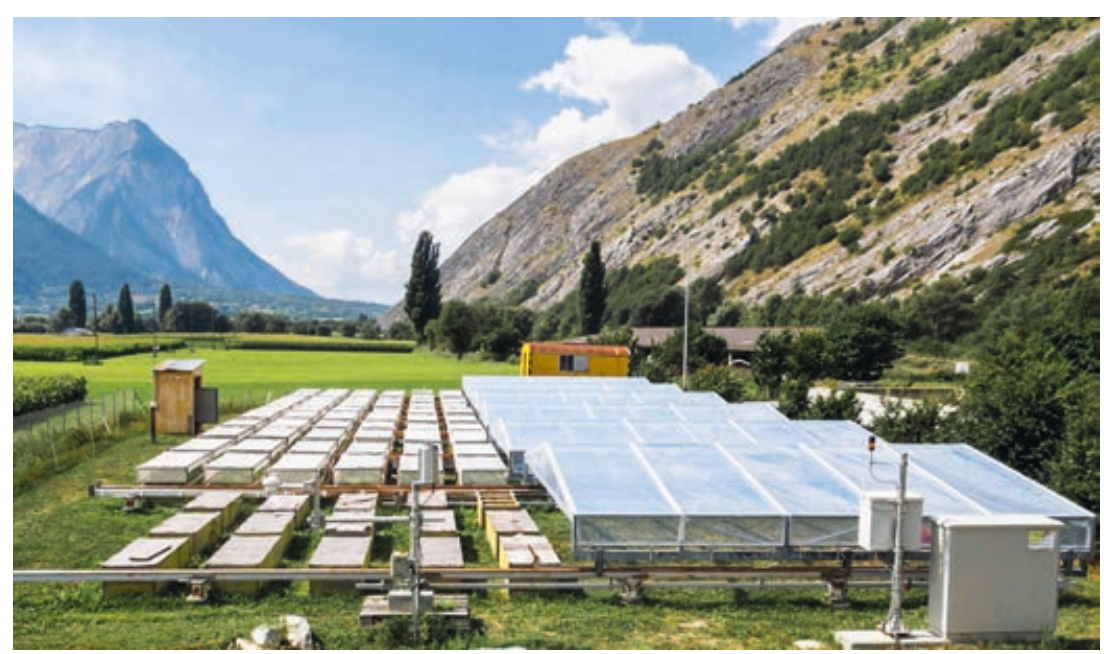

Abb 1 Regendachanlage am Fuss der Walliser Felsensteppe in der Nähe von Leuk. Foto: Christoph Bachofen

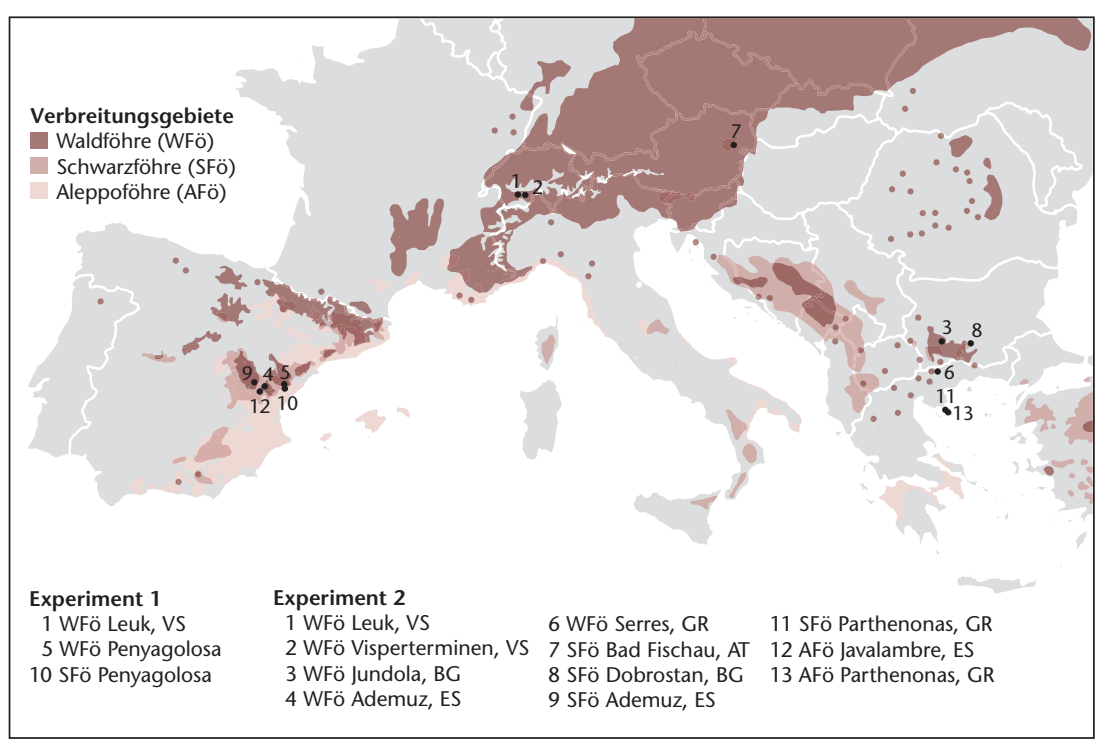

Abb 2 Herkunft des Saatguts. Verbreitungskarten von www.eurforgen.org. mehrere Generationen erfolgt (Aitken et al 2008). Gerade in den Zentralalpen, wo der Niederschlag von Jahr zu Jahr stark variiert, müssen Sämlinge nebst Trockenheitstoleranz auch eine ausreichende phänotypische Plastizität aufweisen, damit sie trockene Jahre überleben und gleichzeitig in klimatisch günstigen Jahren schnell wachsen können, um konkurrenzstark zu sein (Moser et al 2015).

Um der Frage nachzugehen, wie sich ein trockeneres Klima auf die Waldföhrenverjüngung in den Tieflagen der Zentralalpen auswirken könnte, haben wir in verschiedenen Garten- und Feldexperimenten untersucht, wie variabel die heimische Waldföhre während der Verjüngungsphase auf Umweltänderungen, insbesondere Temperatur und Trockenheit, reagiert. Im ersten Experiment, das 2009 in der Nähe von Leuk (VS) stattfand, lag der Schwerpunkt auf der Keimungsphase und den Fragen, 1) wie stark sich höhere Temperaturen zusammen mit Frühjahrs- und/oder Sommertrockenheit auf die Keimung und das Überleben junger Waldföhren auswirken, 2) wie anpassungsfähig (plastisch) die lokalen Herkünfte in Bezug auf Trockenheit sind, und 3) ob Waldföhrenherkünfte vom südlichen Verbreitungsrand der Art in Spanien oder die nah verwandte Schwarzföhre ( $P$. nigra) besser an das zukünftige Klima der Zentralalpen angepasst sind (Richter et al 2012). In einem zweiten Experiment (2012-2014) wurde die Auswahl an mediterranen Waldföhrenherkünften erweitert und neben der Schwarzföhre auch die Aleppoföhre (P. halepensis) einbezogen (Bachofen et al 2018). Dieser Artikel basiert auf diesen in Englisch erschienenen Originalbeiträgen.

\section{Material und Methoden}

\section{Versuchsstandort und experimenteller Aufbau}

Die beiden Gartenexperimente fanden in der Regendachanlage der Eidgenössischen Forschungsanstalt WSL am Fuss der Felsensteppe im Leukerfeld bei Leuk statt (Abbildung 1). Dort wurden Samen verschiedener Föhrenarten und -herkünfte in Mesokosmen (kleine, vereinfachte Ökosysteme) ausgesät. Die Samen stammten nicht von anerkannten Samenerntebeständen, sondern von Einzelbaumernten in autochthonen Beständen an möglichst trockenen Standorten (Abbildung 2). Die Mesokosmen wurden je nach Experiment unterschiedlichen Umweltbedingungen (Temperatur, Bewässerung, Beschattung) ausgesetzt (Tabelle 1). Sie waren $60 \mathrm{~cm}$ tief und enthielten einen zweischichtigen Boden aus $45 \mathrm{~cm}$ Rhonekies und $12 \mathrm{~cm}$ Waldhumus (Experiment 1) oder Gartenerde (Experiment 2). Dieser Aufbau sollte die flachgründigen, steinigen Böden der südexponierten Lagen des Rhonetals nachahmen (Details s. Richter et al 2012, Bachofen et al 


\begin{tabular}{|c|c|c|}
\hline Faktoren & $\begin{array}{l}\text { Experiment } 1 \\
\text { (Keimungsphase) }\end{array}$ & $\begin{array}{l}\text { Experiment } 2 \\
\text { (Sämlingsphase) }\end{array}$ \\
\hline Temperatur & $\begin{array}{l}\text { 1) Umgebungstemperatur } \\
\text { 2) }+2.5^{\circ} \mathrm{C} \\
\text { 3) }+5^{\circ} \mathrm{C}\end{array}$ & 1) Umgebungstemperatur \\
\hline $\begin{array}{l}\text { Bewässerung } \\
\text { (Mitte März } \\
\text { bis Mitte } \\
\text { September) }\end{array}$ & $\begin{array}{l}\text { 1) Feucht (Abbildung } 5 a \text { ) } \\
\text { 2) Frühjahrs- und Sommer- } \\
\text { trockenheit (Abbildung 5b) } \\
\text { 3) Sommertrockenheit } \\
\text { (Abbildung 5c) }\end{array}$ & $\begin{array}{l}\text { 1) Feucht (Abbildung } 5 d \text { ) } \\
\text { 2) Frühjahrs- und Sommer- } \\
\text { trockenheit (Abbildung } 5 e \text { ) } \\
\text { 3) Sommerdürre (Abbildung } 5 f \text { ) }\end{array}$ \\
\hline Licht & 1) $100 \%$ Sonnenlicht & 1) $78 \%$ Sonnenlicht \\
\hline
\end{tabular}

Tab 1 Untersuchte Umweltbedingungen in Experiment 1 (2009) und Experiment 2 (2012-2014). Details zu den gegossenen Wassermengen siehe Abbildung 5.

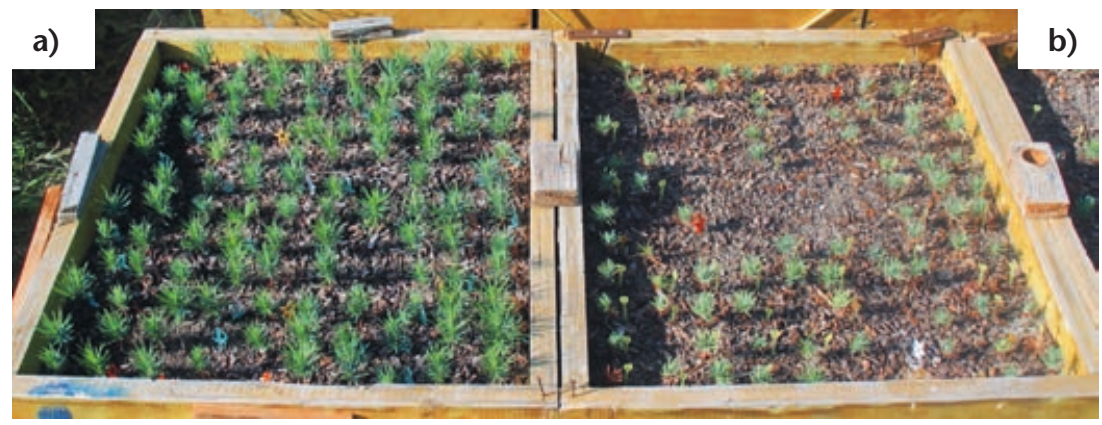

Abb 3 Experiment 1 (Keimungsphase): Mesokosmen $(0.5 \mathrm{~m} \times 0.6 \mathrm{~m})$ mit sechs Monate alten Wald- und Schwarzföhren unterschiedlicher Herkunft (Abbildung 2). Jeder Mesokosmos enthält 120 Keimlinge (40 pro Art und Herkunft), wobei Arten und Herkünfte zufällig angeordnet sind. Bewässerungsvarianten: a) feucht (432 mm von Mitte März bis Mitte September 2009); b) Frühjahrs- und Sommertrockenheit (218 mm). Foto: Thomas Wohlgemuth
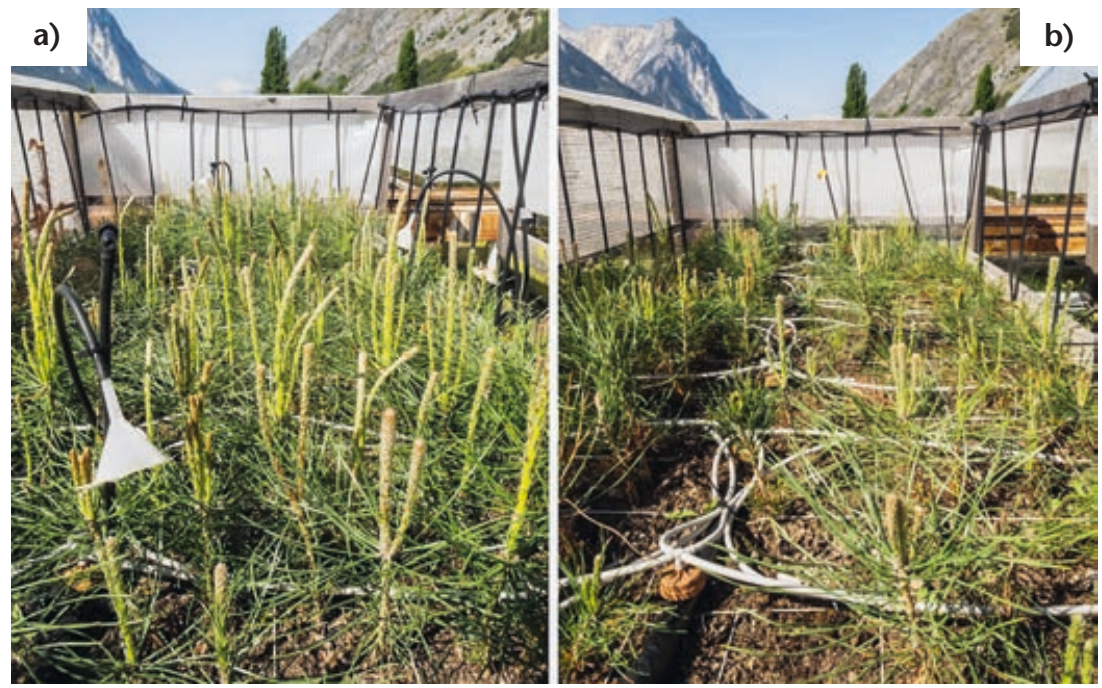

Abb 4 Experiment 2 (Sämlingsphase): Mesokosmos $(0.8 \mathrm{~m} \times 2.0 \mathrm{~m}$ ) mit 2-jährigen Wald-, Schwarz- und Aleppoföhren unterschiedlicher Herkunft (Abbildung 2). Jeder Mesokosmos enthält 140 Sämlinge (10 pro Art und Herkunft), die zufällig angeordnet sind. Die Mesokosmen wurden mit einem Tropfbewässerungssystem (weisse Schläuche) automatisch bewässert und mit einem weissen Tuch beschattet (das obere Beschattungstuch wurde für die Aufnahme entfernt). Bewässerungsvarianten: a) feucht (432 mm von Mitte März bis Mitte September), b) extreme Sommertrockenheit (160 mm). Fotos: Christoph Bachofen

2018). In Experiment 1 waren die Mesokosmen der direkten Sonnenstrahlung ausgesetzt (Abbildung 3), was den Keimungs- und Wachstumsbedingungen in Waldöffnungen oder auf einer Waldbrandfläche entspricht, während sie in Experiment 2 mit Beschattungstüchern versehen waren, die $22 \%$ des
Sonnenlichts abschirmten (Abbildung 4). Die Regendachanlage umfasste sechs mobile Dächer, die sich bei Regen automatisch über die Mesokosmen bewegten und nach dem Regen wieder in die Ausgangsstellung zurückrollten (Abbildung 1). Dies erlaubte uns, die Waldföhren von der Samenkeimung bis zum Alter von 3 Jahren unter den natürlichen klimatischen Gegebenheiten (Sonneneinstrahlung, Temperatur, Wind usw.) des Rhonetals aufzuziehen und gleichzeitig den Niederschlag zu kontrollieren. Dazu wurde wöchentlich in zwei aufeinanderfolgenden Nächten von Hand (Experiment 1) bzw. mithilfe eines Tropfbewässerungssystems (Experiment 2; $\mathrm{Ab}$ bildung 4) die gewünschte Wassermenge gegossen. In beiden Experimenten wurden drei Bewässerungsvarianten verglichen: 1) feuchtes Frühjahr (März bis Mai) und feuchter Sommer (Juni bis September), 2) feuchtes Frühjahr, gefolgt von Sommertrockenheit (mediterrane Verhältnisse), 3) Frühjahrs- und Sommertrockenheit (zentralalpine Verhältnisse). Die Wassermengen in den Varianten 2 und 3 waren in Experiment 1 höher als in Experiment 2 (Details s. Abbildung 5). In Variante 2 wurden die Mesokosmen in Experiment 2 während der Sommermonate gar nicht bewässert (4-monatige Sommerdürre; $\mathrm{Ab}$ bildung 5f). Zusätzlich zu den verschiedenen Bewässerungsvarianten wurde in Experiment 1 mithilfe von Thermokabeln, die $0.5 \mathrm{~cm}$ unter der Bodenoberfläche vergraben waren, die Bodentemperatur um $+0{ }^{\circ} \mathrm{C}$ (Umgebungstemperatur), $+2.5{ }^{\circ} \mathrm{C}$ oder $+5{ }^{\circ} \mathrm{C}$ erhöht (Details s. Richter et al 2012).

Die Föhrensamen wurden in beiden Experimenten Mitte März direkt in die Mesokosmen gesät. In Experiment 1 wurden die Mesokosmen gleich nach der Aussaat unterschiedlich bewässert, sodass der Effekt des Bewässerungsregimes auf Keimung und Keimlingsmortalität untersucht werden konnte. Dazu wurden die gekeimten Samen während der ersten zwei Monate alle ein bis drei Tage gezählt und markiert. Die Mortalität der Keimlinge wurde zuerst zweiwöchentlich, ab Juli nur noch monatlich festgehalten. In Experiment 2 stand das Sämlingswachstum im Vordergrund, weshalb von der Aussaat bis Ende Mai alle Mesokosmen ausgiebig bewässert wurden, um in allen Behandlungsvarianten vergleichbare Keimungs- und Mortalitätsraten zu erreichen.

Am Ende der ersten Vegetationsperiode (Experiment 1) bzw. der zweiten und dritten (Experiment 2) wurde jeweils bei zufällig ausgewählten Keimlingen/Sämlingen die Höhe und die oberirdische Biomasse (Trockengewicht) bestimmt. Bei ausgewählten Behandlungskombinationen wurden auch die Wurzeln ausgegraben und vermessen. In Experiment 1 wurden die Wurzeln von 75 Waldföhrenkeimlingen der Leuker Herkunft genauer untersucht: Mithilfe der Software WinRhizo (Regent Instruments Inc., Canada) wurde die kumulative Wurzellänge gemessen, und unter dem Mikroskop 
Experiment 1
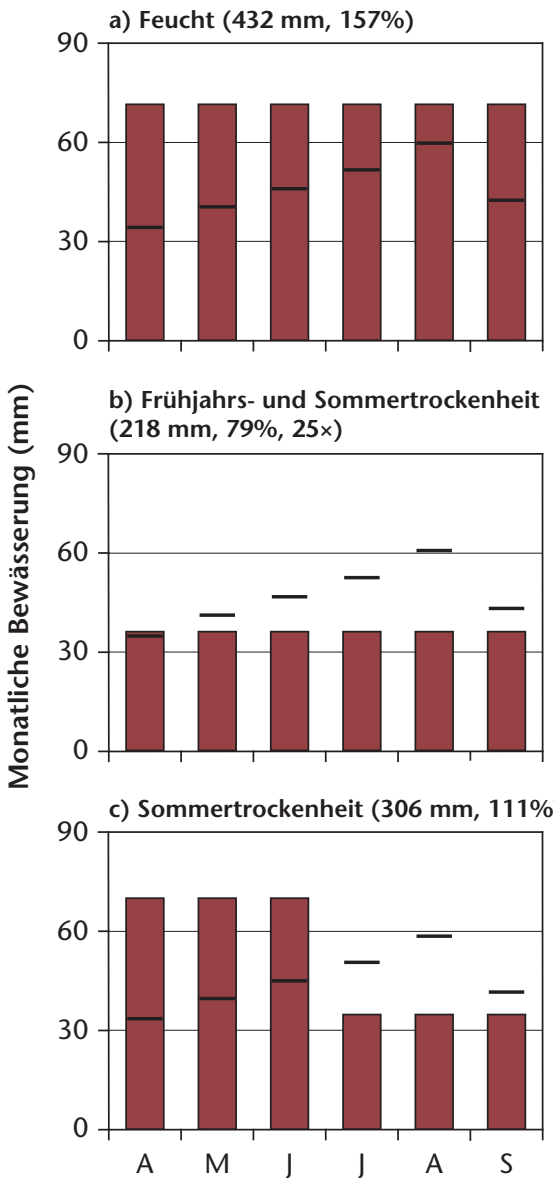

Experiment 2

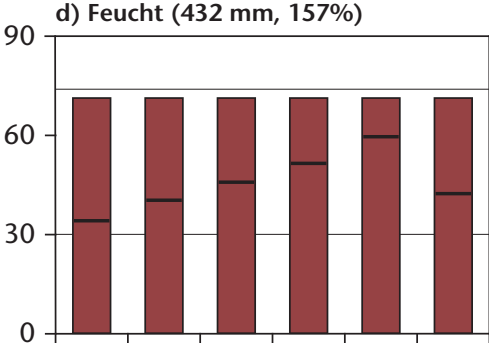

e) Frühjahrs- und Sommertrockenheit (189 mm, 68\%, 12x)
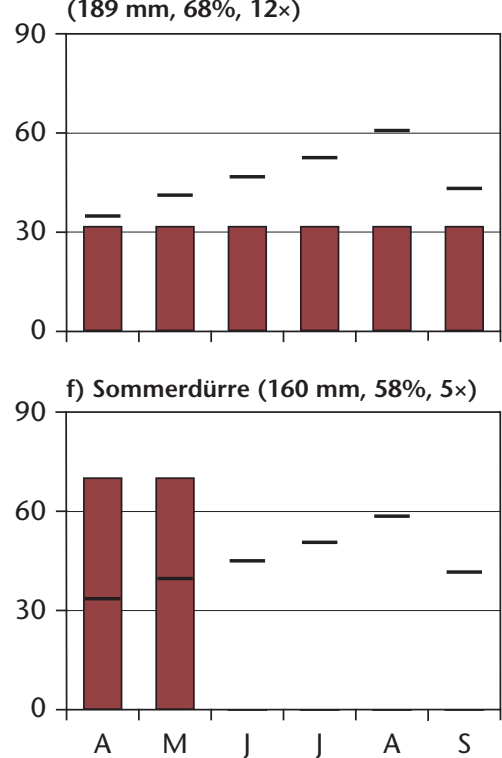

wurde die Anzahl Wurzelspitzen gezählt (Details s. Moser et al 2015).

\section{Datenauswertung}

Die Effekte von Temperatur, Bewässerung und Samenherkunft auf Keimung, Überleben und Wachstum der Pflanzen wurden mithilfe von gemischten linearen Modellen auf Signifikanz geprüft (R-Statistik-Pakete «lme4» und «lmerTest»). Die Klimadaten der Samenherkunftsorte stammen von MeteoSchweiz (Leuk, Visperterminen) und von worldclim. org (übrige Herkünfte), wobei die Temperatur- und Niederschlagsangaben über den Zeitraum von 1950 bis 2000 gemittelt wurden. Die klimatische Wasserbilanz am Herkunftsort (Differenz zwischen Niederschlag und potenzieller Verdunstung) wurde nach Thornthwaite (1948) berechnet.

Die phänotypische Plastizität einer Art bzw. einer Population/Herkunft wurde mit dem Relative Distance Plasticity Index (RDPI) von Valladares et al (2006) berechnet, der zwischen 0 (keine Plastizität) und 1 (hohe Plastizität) variiert. Der Index beruht auf der Differenz eines Merkmals zwischen genetisch ähnlichen Individuen (hier: Herkünfte/Populationen), die unter verschiedenen Umweltbedingungen (hier: feucht, Frühjahrs- und Sommertrockenheit, extreme Sommertrockenheit) gewachsen sind.

\section{Resultate}

\section{Keimung und Überlebensrate}

Die ersten Keimlinge kamen Anfang April, 21 Tage nach der Aussaat, zum Vorschein, und 90\% der Keimung fanden in den darauffolgenden 10 Ta-
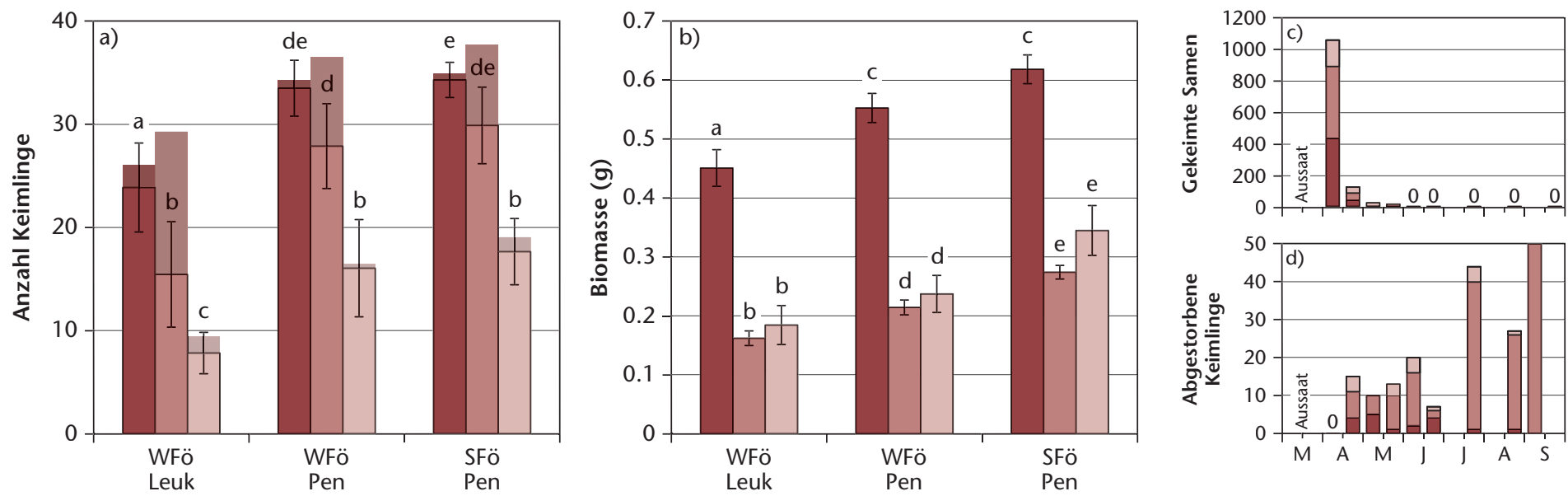

Anzahl Tot

gekeimter
Samen

Bewässerungsregime

$\square$ Feucht $\square$ Sommertrockenheit $\square$ Frühjahrs- und Sommertrockenheit

Abb 6 Experiment 1 (Keimungsphase): a) Anzahl gekeimte von 40 ausgesäten Samen (gesamter Balken), Anzahl abgestorbene Keimlinge (farbiger Balkenteil oberhalb des umrandeten Teils) sowie Anzahl lebende Keimlinge (schwarz umrandeter Balkenteil \pm Standardfehler) pro Mesokosmos und b) deren Biomasse (Wurzel und Spross; Mittelwert \pm Standardfehler) am Ende der ersten Vegetationsperiode in Abhängigkeit von Bewässerungsregime, Baumart (WFö: Waldföhre; SFö: Schwarzföhre) und Samenherkunft (Leu: Leuk, VS; Pen: Penyagolosa, Spanien). c) und d) Saisonaler Verlauf von Samenkeimung und Keimlings-

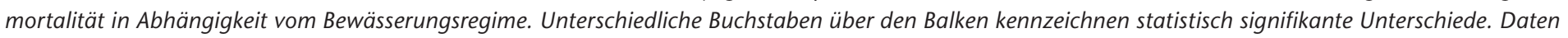
aus Richter et al (2012). 

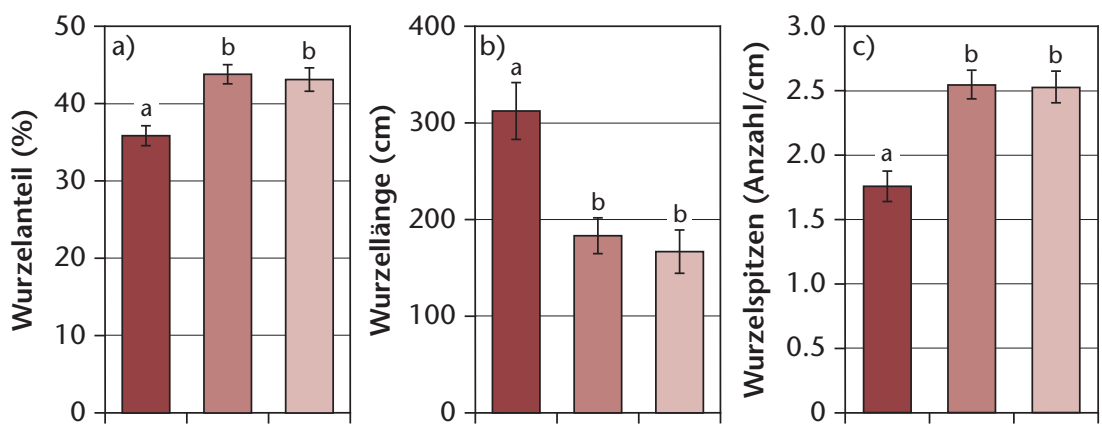

Bewässerungsregime

$\square$ Feucht $\square$ Sommertrockenheit $\square$ Frühjahrs- und Sommertrockenheit

Abb 7 Experiment 1 (Keimungsphase): a) Wurzelanteil an der Gesamtbiomasse, b) Wurzellänge und c) Anzahl Wurzelspitzen pro Wurzellänge bei unterschiedlich bewässerten Waldföhrenkeimlingen aus Leuk, VS, sechs Monate nach der Keimung (Mittelwert \pm Standardfehler). Unterschiedliche Buchstaben über den Balken kennzeichnen statistisch signifikante Unterschiede. Daten aus Moser et al (2015).

gen statt (Abbildung 6c). Die Keimungsrate und das Überleben der Waldföhre während der ersten Vegetationsperiode waren primär von der Bewässerung und in geringerem Masse von der Bodentemperatur abhängig (Keimungsrate, Varianzanalyse: Effektgrösse $\eta^{2}$ Bewässerung $=0.875, \eta^{2}$ Bodentemperatur $=0.285$; Überlebensrate, verallgemeinertes lineares Modell: Regressionskoeffizienten $\beta_{\text {sommertrockenheit }}=-2.343, \beta_{+5^{\circ} \mathrm{C}}=-1.074$; Details s. Richter et al 2012). Im Vergleich zur feuchten Bewässerungsvariante reduzierte die Frühjahrstrockenheit in der Bewässerungsvariante «Frühjahrsund Sommertrockenheit» (Abbildung 5b) die Keimungsrate der Leuker Waldföhren um rund einen Drittel, und die kombinierte Frühjahrs- und Sommer- trockenheit führte prozentual gesehen zu einer fünfmal höheren Keimlingsmortalität (Abbildung 6a). Damit waren am Ende der Vegetationsperiode in den feuchten Mesokosmen dreimal mehr Keimlinge vorhanden und diese hatten 2.5-mal mehr Biomasse als in den Mesokosmen mit kombinierter Frühjahrs- und Sommertrockenheit (Abbildungen 6a und 6b). Unter Niederschlagsbedingungen, wie sie in mediterranen Gebieten herrschen, d.h. bei simulierten hohen Niederschlägen im Frühjahr und Trockenheit im Sommer (Abbildung 5c), war die Keimungsrate gleich hoch wie unter feuchten Bedingungen (Abbildung 6a). Eine deutlich erhöhte Sterberate während der Sommertrockenheit (Juli bis September; Abbildungen 6a und 6d) führte dazu, dass am Ende der ersten Vegetationsperiode weniger lebende Waldföhren vorhanden waren als unter feuchten Bedingungen, jedoch deutlich mehr als bei kombinierter Frühjahrs- und Sommertrockenheit (Abbildung 6a). Bei allen Bewässerungsvarianten war die Hälfte der Keimlingsmortalität im Frühjahr (April bis Mai) auf die Umfallkrankheit (ausgelöst durch verschiedene Pilze wie Fusarium sp. oder Botrytis sp.) und nicht auf Trockenheit zurückzuführen.

\section{Akklimatisation}

Die Waldföhrenkeimlinge zeigten in den ersten Monaten nach der Keimung (Experiment 1) ein erstaunliches Anpassungspotenzial. Im Vergleich zur feuchten Bewässerungsvariante investierten sie bei kombinierter Frühjahrs- und Sommertrockenheit deutlich mehr ins Wurzelwachstum als ins Sprosswachstum (Abbildung 7a). Absolut gemessen waren
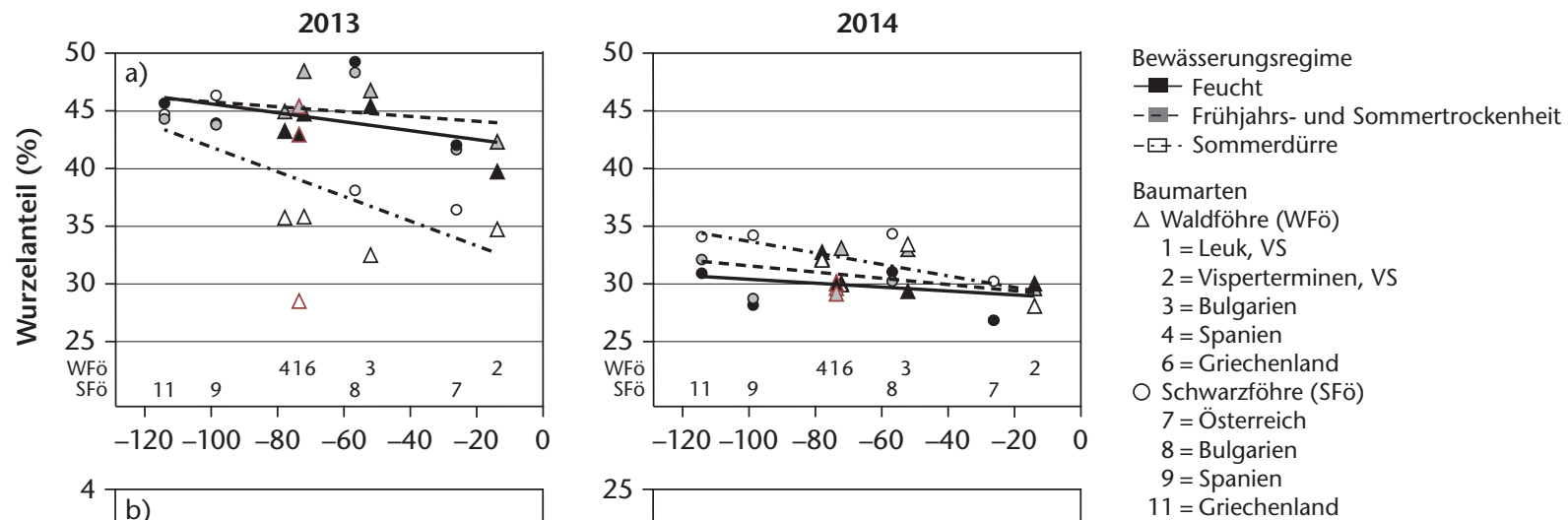

1 = Griechenland
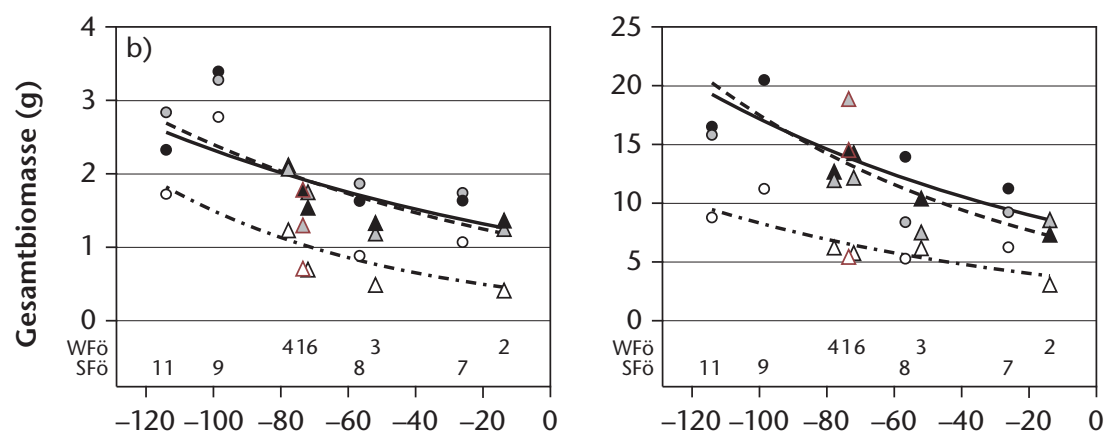

Klimatische Wasserbilanz Juli (mm)

Klimatische Wasserbilanz Juli $(\mathrm{mm})$

Abb 8 Experiment 2 (Sämlingsphase): a) Wurzelanteil an der Gesamtbiomasse und b) Gesamtbiomasse (Wurzel und Spross) von zwei- und dreijährigen Wald- und Schwarzföhrensämlingen in Abhängigkeit vom Bewässerungsregime und von der klimatischen Juli-Wasserbilanz am Herkunftsort. Die Walliser Tieflagenherkunft (1) ist rot markiert. Daten aus Bachofen et al (2018). 
a) Waldföhre
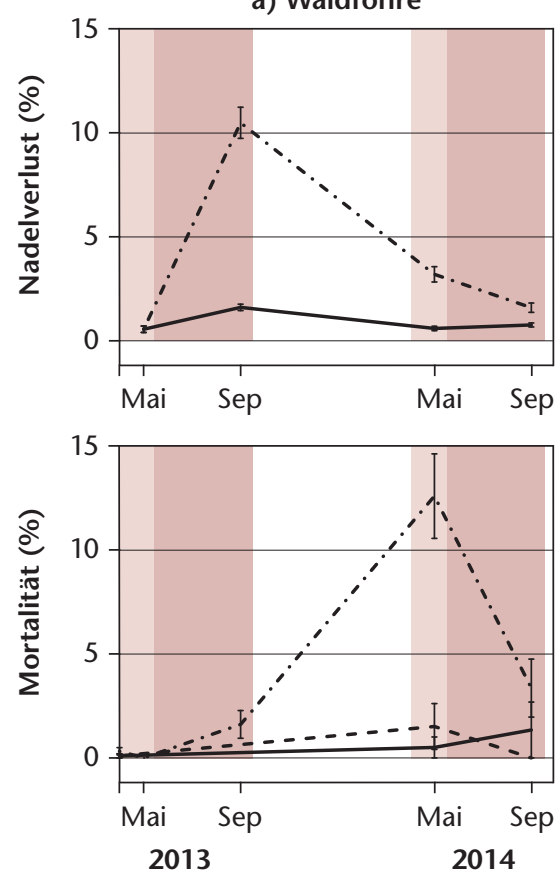

2013 b) Schwarzföhre

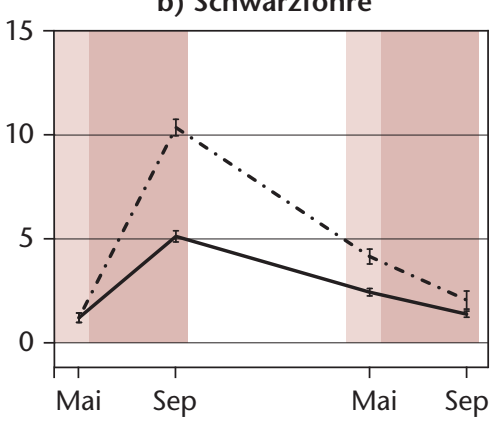$$
15
$$

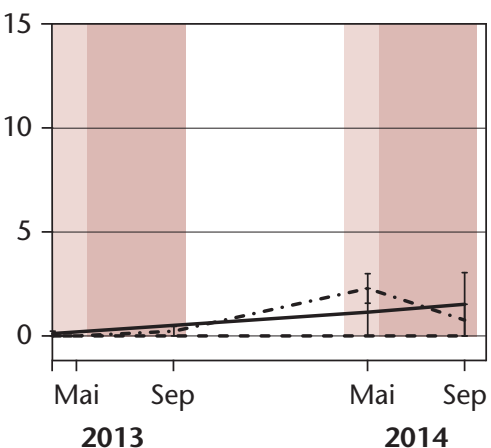

Bewässerungsregime

— Feucht - - - Frühjahrs- und Sommertrockenheit - -.- Sommerdürre

Abb 9 Experiment 2 (Sämlingsphase): Nadelbiomassenverlust und Sämlingsmortalität (Mittelwert \pm Standardfehler) von zwei- und dreijährigen a) Wald-und b) Schwarzföhren in unterschiedlich bewässerten Mesokosmen gemessen jeweils im Frühjahr und im Herbst. Der Nadelbiomassenverlust wurde nur in den Mesokosmen mit feuchtem Bewässerungsregime und denjenigen mit Sommerdürre geschätzt. Die hellroten Flächen kennzeichnen den Zeitraum der Frühjahrstrockenheit, die dunkelroten denjenigen der Sommertrockenheit bzw. Sommerdürre.

a) Einzelne Merkmale

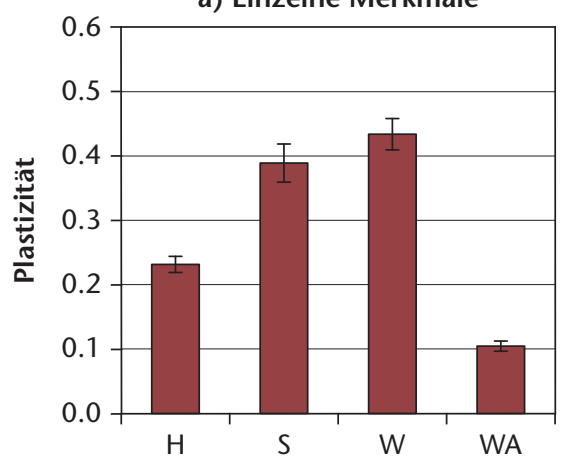

b) Wurzelanteil

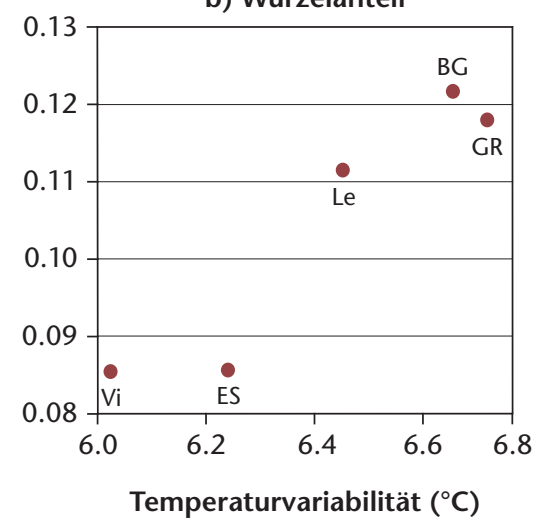

Abb 10 Experiment 2 (Sämlingsphase): Phänotypische Plastizität von dreijährigen Waldföhren aus dem Wallis und dem Mittelmeergebiet. a) Plastizität einzelner Merkmale (Mittelwert der Provenienzen \pm Standardfehler): Pflanzenhöhe $(H)$, Spross- und Wurzelbiomasse (S bzw. W) sowie Wurzelanteil (WA); b) Plastizität des Wurzelanteils innerhalb einer Population in Abhängigkeit von der Variabilität der Monatsmitteltemperaturen am Ursprungsort (Le=Leuk, VS; Vi=Visperterminen, VS; $B G=J u n d o l a, B u l g a r i e n ; ~ E S=A d e m u z$, Spanien; $G R=$ Serres, Griechenland). Der Plastizitätsindex schwankt zwischen 0 (keine Plastizität) und 1 (hohe Plastizität); Klimadaten 1950 bis 2000 von www.worldclim.org.

die Wurzeln der trockengestressten Keimlinge zwar nur halb so lang wie diejenigen der nicht gestressten (Abbildung 7b), sie bildeten jedoch mehr Wurzelspitzen pro Wurzellänge, was die Kapazität zur Wasserund Nährstoffaufnahme erhöht (Abbildung 7c). Überraschenderweise hatten die Keimlinge, die erst ab Juni der Trockenheit ausgesetzt waren (Sommer-

trockenheit), am Ende der Vegetationsperiode den gleichen Wurzelanteil, die gleiche Wurzellänge und die gleiche Wurzelspitzendichte wie die bereits im Frühjahr trockengestressten Waldföhren (Frühjahrsund Sommertrockenheit; Abbildung 7). Die Keimlinge waren somit in der Lage, ihren Wurzelanteil innerhalb derselben Vegetationsperiode an die plötzlich einsetzende Sommertrockenheit anzupassen.

Diese kurzfristige Anpassungsfähigkeit war in der zweiten Vegetationsperiode bei den Sämlingen in Experiment 2 nicht mehr vorhanden: Waldföhren, die bis Ende Mai des zweiten Jahres unter feuchten Bedingungen aufwuchsen und dann extremer Sommertrockenheit (Sommerdürre) ausgesetzt waren, hatten einen deutlich geringeren Wurzelanteil als Sämlinge in der feuchten Bewässerungsvariante (Abbildung 8a). Die Sommerdürre führte zu vermehrtem Absterben von Nadelbiomasse und einer erhöhten Sterberate im Anschluss an die Trockenheit (Abbildung 9a). Auch wenn die Sämlinge kurzfristig nicht reagieren konnten, zeigte sich ihr Anpassungspotenzial in der nächsten Vegetationsperiode. Im dritten Jahr litten sie kaum mehr unter erneuter Sommerdürre. Sie hatten nun den gleichen Wurzelanteil (Abbildung 8a) und nur einen geringfügig höheren Nadelverlust als die Sämlinge der feuchten Bewässerungsvariante (Abbildung 9a).

\section{Plastizität und lokale Anpassung}

Wie im vorherigen Abschnitt beschrieben, passten die Waldföhren ihr Erscheinungsbild (Phänotyp) den herrschenden Umweltbedingungen an, zum Beispiel indem Keimlinge bei Trockenheit mehr Wurzeln im Verhältnis zum Spross entwickelten (Abbildung 6a). Die phänotypische Plastizität war nicht bei allen Pflanzenmerkmalen gleich ausgeprägt: Während Spross- und Wurzelbiomasse im Sämlingsalter eine hohe Plastizität aufwiesen, unterschied sich der Wurzelanteil bei den dreijährigen Waldföhren nur wenig zwischen den Bewässerungsvarianten (Abbildung 10a). Stattdessen war die phänotypische Plastizität des Wurzelanteils korreliert mit dem Klima am Herkunftsort der Samen: Je grösser die klimatische Variabilität am Ursprungsort (Standardabweichung der Monatsmitteltemperaturen) war, desto plastischer war der Wurzelanteil (Abbildung 10b). Auch bei der Biomasse war ein Zusammenhang mit dem Herkunftsklima erkennbar: Die Sämlinge aus Gebieten mit ausgeprägter Sommertrockenheit waren deutlich schwerer als jene aus Gebieten, in denen das klimatische Wasserdefizit im Juli klein und damit die Gefahr der Austrocknung im Sommer gering ist (Abbildung 8b).

\section{Nicht heimische Baumarten}

Die aus dem Mittelmeergebiet stammende Schwarzföhre war sowohl im Keimlings- als auch im Sämlingsalter etwas trockenresistenter als die ein- 
heimische Waldföhre aus dem Wallis. Unter feuchten wie auch unter trockenen Bedingungen waren Keimungsrate, Anzahl überlebende Keimlinge und deren Biomasse am Ende der ersten Vegetationsperiode leicht höher als bei den Leuker Waldföhren, jedoch etwa gleich gross wie bei den spanischen Waldföhren (Abbildung 6). Dieser Grössenunterschied blieb auch später bestehen (Abbildung 8b). Obwohl der Nadelbiomassenverlust nach der ersten extremen Sommertrockenheit ähnlich hoch war wie bei der Waldföhre, starben im Anschluss an die Sommerdürre deutlich weniger Schwarzföhren ab (Abbildung 9b). Auch die Aleppoföhre etablierte sich in der ersten Vegetationsperiode erfolgreich. Allerdings überlebten nur wenige Sämlinge den ersten Winter, sodass wir diese Art im zweiten und dritten Versuchsjahr nicht weiter untersuchen konnten.

\section{Diskussion}

Damit Samen keimen und Sämlinge anwachsen können, sind feuchte Witterungsbedingungen erforderlich. Natürliche Verjüngung findet deshalb pulsartig, das heisst in begrenzten Zeitfenstern statt (Grubb 1977, Moser et al 2015). Dies trifft insbesondere auf trockene Regionen (Zackrisson et al 1995, Savage et al 1996) und Orte mit limitierter Vegetationszeit zu (Ott et al 1991). Es wird davon ausgegangen, dass diese günstigen Zeitfenster in Zukunft bei steigenden Temperaturen und sinkenden Sommerniederschlägen kürzer und seltener werden. In den Tieflagen des Wallis, wo bereits heute ein trockenes Klima herrscht, könnte dies besonders nach grossflächigen Waldbränden die Waldföhrenverjüngung erschweren, weil sich diese Art nur über Samen und nicht durch Stockausschläge verjüngen kann (s. auch Wohlgemuth et al 2018, dieses Heft). Eine erfolgreiche Verjüngung hängt in der ersten Phase von Keimungsrate und Mortalität $a b$, wobei die Gartenexperimente in der Regendachanlage bei Leuk gezeigt haben, dass eine hohe Keimungsrate für den erfolgreichen Anwuchs der Waldföhre in Trockenregionen entscheidend ist. Fehlende Frühjahrsniederschläge beeinträchtigten die Keimungsrate stärker, als dass Sommertrockenheit die Überlebensrate reduzierte (Abbildung 6). Damit war die Anzahl überlebender Keimlinge am Ende der Vegetationsperiode bei kombinierter Frühjahrs- und Sommertrockenheit deutlich geringer als in den Bewässerungsvarianten «feucht» und «Sommertrockenheit», obschon die Keimlinge in der Bewässerungsvariante «Frühjahrsund Sommertrockenheit» die Sommertrockenheit deutlich häufiger überlebten als die Keimlinge, die ein feuchtes Frühjahr erlebt hatten (Abbildung 6d). Im Gegensatz zur Trockenheit schmälerten höhere Temperaturen Keimungsrate und Keimlingswachstum nur geringfügig (Richter et al 2012). Interessan- terweise wirkte sich ein Anstieg der Bodentemperatur um $+5{ }^{\circ} \mathrm{C}$ weniger negativ auf die Überlebensrate der Keimlinge aus als ein Anstieg um $+2.5^{\circ} \mathrm{C}$ : Die Samen keimten bei der höheren Temperatur früher, wodurch sich die Überlebensrate der Keimlinge geringfügig erhöhte (Richter et al 2012). Dass Keimung und Etablierung von Nadelbäumen primär von den Frühjahrsniederschlägen abhängen, haben auch Feldexperimente im Churer Rheintal gezeigt (Moser et al 2015). Gartenexperimente können somit durchaus praxisrelevante Hinweise zum Verhalten von Baumkeimlingen liefern, selbst wenn die Umweltbedingungen in den Mesokosmen nur bedingt denjenigen in freier Natur entsprechen. Dass Frühjahrsniederschläge für den Anwuchs der Waldföhre entscheidend sind, ist im Hinblick auf den Klimawandel insofern als positiv zu bewerten, als sich die Frühjahrsniederschläge bis 2085 nur wenig ändern sollen (C2SM et al 2011).

\section{Akklimatisation}

Wo Pflanzen die durch Fotosynthese gewonnenen Assimilate einsetzen, um unter den herrschenden Umweltbedingungen konkurrenzfähig zu sein, zählt zu den faszinierendsten Fragen der Pflanzenökologie (z.B. Fridley 2017). Pflanzen müssen verschiedene Ziele gegeneinander abwägen: Ein schnelles Höhenwachstum ermöglicht es den Keimlingen/ Sämlingen, sich im Konkurrenzkampf um Licht gegenüber anderen Pflanzen zu behaupten, mit grosser Blattmasse können sie viel Kohlenstoff fixieren, ein ausgedehntes Wurzelwerk sichert während längerer Trockenphasen den Zugang zu Wasser und Nährstoffen. Bei Waldföhrenkeimlingen scheint der Wurzelanteil ein wichtiger Faktor zur Akklimatisation an Trockenstress zu sein. Unabhängig davon, ob die Trockenheit bereits im Frühjahr oder erst im Sommer einsetzte, hatten die trockengestressten Keimlinge am Ende der ersten Vegetationsperiode einen grösseren Wurzelanteil als Keimlinge in der feuchten Bewässerungsvariante, auch wenn die absolute Wurzellänge deutlich kleiner war (Abbildung 7). Durch eine höhere Dichte von Wurzelspitzen pro Wurzellänge konnten die Pflanzen einen Teil der fehlenden Wurzellänge kompensieren und damit vermutlich die Aufnahmekapazität von Wasser- und Nährstoffen erhöhen. In der zweiten Vegetationsperiode geschah die Anpassung an Trockenheit nicht mehr über eine Änderung des Wurzelanteils. Dieser war unter feuchten Bedingungen und bei kombinierter Frühjahrs- und Sommertrockenheit gleich, während sich der Wurzelanteil wohl als Folge der Sommerdürre sogar reduzierte (Abbildung 8a). Auf den ersten Blick mag diese Reduktion erstaunen, da sie der allgemeinen Annahme zuwiderläuft, dass Pflanzen ihre Biomasse in dasjenige Organ investieren, das die am stärksten limitierte(n) Ressource(n) erschliesst («optimal partitioning theory») - bei Trockenheit 
also die Wurzeln, die für die Wasseraufnahme verantwortlich sind (McCarthy \& Enquist 2007). Wir erklären uns den kleineren Wurzelanteil damit, dass das Sprosswachstum bei der Waldföhre vor dem Wurzelwachstum stattfindet (Iivonen et al 2001). Das bedeutet, dass die Sämlinge unter den optimalen Bedingungen im Frühjahr grosse Sprosse ausbildeten, jedoch später wegen der Sommerdürre nicht mehr genügend Ressourcen für das Wurzelwachstum hatten. Somit konnten sie ihr plötzlich ungünstiges Wurzel-Spross-Verhältnis nicht mehr ausgleichen. Dass ein kleiner Wurzelanteil während einer Dürreperiode von Nachteil ist, zeigt sich im hohen Nadelbiomassenverlust und der erhöhten Sterberate nach der Dürre im zweiten Jahr (Abbildung 9). Im dritten Jahr hat der Wurzelanteil dann generell abgenommen, was der natürlichen Entwicklung (Ontogenie) von Pflanzen entspricht (Poorter et al 2015). Die Waldföhrensämlinge hatten sich bis dahin jedoch anderweitig akklimatisiert, zum Beispiel durch die Bildung kürzerer Nadeln (Perret-Gentil 2016). Damit verringert sich die Verdunstung (Transpiration) und entsprechend der Wasserbedarf der ganzen Pflanze, sodass eine weitere Dürreperiode weder zu Nadelverlust noch zu Mortalität führte. Dass mehr als 85\% der Waldföhrensämlinge zwei aufeinanderfolgende Sommerdürren überstanden, hat uns erstaunt. Dies zeigt, wie gross das Akklimatisationspotenzial der Waldföhren im Sämlingsstadium ist, was unter anderem damit zusammenhängen dürfte, dass junge Pflanzen rasch wachsen und von einem Jahr zum nächsten ihre Biomasse vervielfachen. So hatten die Waldföhren am Ende der dritten Vegetationsperiode im Schnitt mehr als achtmal mehr Biomasse als noch am Ende der zweiten Vegetationsperiode. Deshalb können bei Sämlingen morphologische Veränderungen wie kürzere Nadeln den Wasserhaushalt von einem Jahr zum nächsten viel stärker beeinflussen als bei adulten Bäumen mit fünf bis sechs aktiven Nadeljahrgängen.

\section{Plastizität versus lokale Anpassung}

Die Waldföhre ist weltweit die am weitesten verbreitete Föhrenart. In Europa kommt sie vom Mittelmeer bis in den hohen Norden vor. Entsprechend ihrem riesigen Verbreitungsgebiet ist die Art enorm plastisch. Sie kann ihr Erscheinungsbild (Höhe, Nadellänge usw.) den herrschenden Umweltbedingungen anpassen und somit an sehr unterschiedlichen Standorten wachsen, wobei sie als beschränkt schattentolerante Art an produktiven Standorten durch schattenwerfende Arten wie die Buche überwachsen wird. Ihre grosse phänotypische Plastizität ist bereits im Keimlings- und Sämlingsalter vorhanden. In den Tieflagen des Rhonetals ist diese kurzfristige Anpassungsfähigkeit von zentraler Bedeutung, weil die Witterung von Jahr zu Jahr stark variiert. Die Waldföhre ist aber auch genetisch divers, wobei der grösste
Teil der genetischen Vielfalt innerhalb und nicht zwischen Populationen liegt (Mátyás et al 2004). Da Föhrenpollen über weite Distanzen fliegen, ist der genetische Austausch zwischen den Populationen gross, und genetische Anpassungen an lokale Umweltbedingungen sind eher gradueller Natur. Dies hat sich auch in unseren Experimenten gezeigt. So hatten die Sämlinge von Herkünften mit einem trockenen Sommerklima, darunter diejenigen aus den Tieflagen des Rhonetals, in allen Bewässerungsvarianten mehr Biomasse produziert als diejenigen aus Gegenden mit feuchteren Sommern (Abbildung 7b). Die Herkünfte mit sommertrockenem Klima hatten auch einen grösseren Wurzelanteil (Abbildung 8a), was im Einklang steht mit der Theorie der optimalen Ressourcenverteilung. Die phänotypische Plastizität gewisser Pflanzenmerkmale wiederum ist genetisch festgelegt: Bei Herkünften aus Gegenden mit starken saisonalen Temperaturschwankungen (Leuk, Bulgarien, Griechenland) war der Wurzelanteil plastischer als bei solchen aus Gegenden mit einem gleichmässigeren Temperaturverlauf (Visperterminen, Spanien; Abbildung 10). Somit hat die Waldföhre die Fähigkeit, sich sowohl kurz- als auch längerfristig an neue klimatische Gegebenheiten anzupassen. Damit ist die phänotypische Plastizität selbst ebenfalls der lokalen Anpassung unterworfen und somit in verschiedenen Klimaregionen unterschiedlich ausgeprägt.

\section{Nicht heimische Baumarten}

Schwarzföhren aus dem Mittelmeergebiet schnitten in der Keimlingsphase vor allem bezüglich Keimungs- und Überlebensrate besser ab als die Waldföhre (Abbildung 6). Im Sämlingsalter überstanden sie die erste Sommerdürre deutlich häufiger als die Waldföhren (Abbildung 9b). Auch mit Winter- und Spätfrost kamen sie gut zurecht (Bachofen et al 2016). Davon zeugen auch grössere Schwarzföhrenbestände im Wallis, zum Beispiel jene bei Thel oberhalb von Leuk. Allerdings ist auch bekannt, dass die Schwarzföhre nach Hagel oder Trockenheit krankheitsanfällig ist. Nach dem trockenen Sommer 2015 waren in der Westschweiz viele Schwarzföhren vom Erreger des Föhrentriebsterbens (Sphaeropsis sapinea) befallen, was auf flachgründigen, kalkhaltigen Böden, zum Beispiel am Jurasüdfuss ob Biel, zu grösseren Ausfällen führte (Meier et al 2016). Gemäss Literatur soll die Aleppoföhre noch wesentlich trockenresistenter sein als die Schwarzföhre. Allerdings fiel sie in unserem Experiment über Winter grösstenteils der Frosttrocknis zum Opfer, sodass wir keine Erkenntnisse zu ihrer Trockentoleranz im Vergleich zu Wald- und Schwarzföhre gewinnen konnten. Spanische Kollegen raten im Übrigen vom Anbau der Aleppoföhre ab, da sie leicht entflammbar ist und in waldbrandgefährdeten Regionen zur Problemart werden kann. 


\section{Schlussfolgerungen}

Übereinstimmend mit früheren Feldexperimenten an südexponierter Lage im Churer Rheintal (Moser et al 2015) zeigen die Resultate dieser Studie, dass sich die Waldföhre nur in Jahren mit genügend Frühjahrsniederschlägen ansamen und in der Folge etablieren kann, und dass in solchen Jahren die Etablierungswahrscheinlichkeit unabhängig von der Herkunft für alle Samen hoch ist. Folglich spielt die Samenherkunft für die erste Phase der Verjüngung eine vernachlässigbare Rolle. Da es weiterhin ab und zu feuchte Frühjahre geben wird, dürfte sich die Waldföhre bei genügend Licht auch in $\mathrm{Zu}$ kunft natürlich verjüngen. In den Tieflagen des Wallis spielt die phänotypische Plastizität für den langfristigen Verjüngungserfolg eine grössere Rolle als die herkunftsbezogene Trockentoleranz, weil damit v.a. in günstigen Jahren ein schnelles und konkurrenzstarkes Keimlings- und Sämlingswachstum ermöglicht wird. In dieser Hinsicht weisen die heimische Herkunft aus den Tieflagen des Wallis und jene aus Griechenland wahrscheinlich die beste Kombination von Trockentoleranz und hoher Plastizität auf, um sich im Klima der Zentralalpen, das von jährlich stark schwankenden Niederschlägen geprägt ist, auch in Zukunft erfolgreich verjüngen zu können. Im weitgehend hagelfreien Rhonetal könnte die etwas trockentolerantere aber ebenso frostresistente Schwarzföhre in Zukunft eine gute Ergänzung zur heimischen Waldföhre sein, während die mediterrane Aleppoföhre den wahrscheinlich auch in $\mathrm{Zu}$ kunft wiederkehrenden (Spät-)Frösten kaum standhalten wird.

Eingereicht: 9. Januar 2018, akzeptiert (mit Review): 26. April 2018

\section{Dank}

Unser Dank geht an T. Kipfer, S. Richter, E. Schnider und A. Walter, die wesentlich zum Erfolg der zwei Experimente beigetragen haben, $\mathrm{K}$. Egger vom Forstbetrieb Leuk und Umgebung und dem Team der ARA Leuk und Umgebung, die uns bei logistischen und technischen Problemen unterstützt haben, und A. Moser und Team vom WSL-Institut für Schnee und Lawinenforschung in Davos für Konstruktion und Wartung der Regendachanlage. Ebenfalls danken wir H. Bachofen, C. Baumann, H. Ding, E. Schnyder, G. Grun, C. Hester, A. Hollaus, A. Joss, A. Käser, K. Kramer, S. Kreuzer, M. Metslaid, Z. Michalova, J. Müller, A. Perret-Gentil, T. Reich, S. Steinböck, D. Trummer, U. Wasem und E. Wilson für die tatkräftige Mithilfe in Garten und Labor. Bei der Samenernte wurden wir unterstützt von S. Berdos, C. Calderón Guerrero, G. Golesch, B. Kinigadner, I. Latchev, A. Tashev und N. Tashev. Finanziert wurden die Projekte vom Schweizerischen National- fonds (Gesuche Nr. 3100A0-118002, 316000-121323, 31003A-140966). Die Klimadaten wurden von MeteoSchweiz zur Verfügung gestellt.

\section{Literatur}

AITKEN SN, YEAMAN S, HOLLIDAY JA, WANG TL, CURTIS MCLANE S (2008) Adaptation, migration or extirpation: climate change outcomes for tree populations. Evol Appl 1: 95111.

BACHOFEN C, WOHLGEMUTH T, GHAZOUL J, MOSER B (2016) Cold temperature extremes during spring do not limit the range shift of Mediterranean pines into regions with intermittent frost. Funct Ecol 30: 856-865.

BACHOFEN C, MOSER B, HOCH G, GHAZOUL J, WOHLGEMUTH T (2018) No carbon 'bet hedging' in pine seedlings under prolonged summer drought and elevated $\mathrm{CO}_{2}$. J Ecol 106: 31-46.

CASTRO J (2006) Short delay in timing of emergence determines establishment success in Pinus sylvestris across microhabitats. Ann Bot 98: 1233-1240.

C2SM, METEOSWISS, ETH, NCCR CLIMATE, OCCC (2011) Swiss climate change scenarios $\mathrm{CH} 2011$. Zürich: C2SM. 88 p.

FRIDLEY JD (2017) Plant energetics and the synthesis of population and ecosystem ecology. J Ecol 105: 95-110.

GRUBB PJ (1977) Maintenance of species-richness in plant communities - importance of regeneration niche. Biol Rev Cambridge Philosophic Soc 52: 107-145.

GUGERLI F, FRANK A, RELLSTAB C, PLUESS AR, MOSER B ET AL (2016) Genetische Variation und lokale Anpassung bei Waldbaumarten im Zeichen des Klimawandels. In: Pluess AR, Augustin S, Brang P, editors. Wald im Klimawandel. Grundlagen für Adaptationsstrategien. Bern: Haupt. pp. 93-113.

IIVONEN S, RIKALA R, VAPAAVUORI E (2001) Seasonal root growth of Scots pine seedlings in relation to shoot phenology, carbohydrate status, and nutrient supply. Can J For Res 31: 1569_ 1578.

MÁTYÁS C, ACKZELL L, SAMUEL CJA (2004) EUFORGEN Technical guidelines for genetic conservation and use for Scots pine (Pinus sylvestris). Rome: International Plant Genetic Resources Institute. $6 \mathrm{p}$

MCCARTHY MC, ENQUIST BJ (2007) Consistency between an allometric approach and optimal partitioning theory in global patterns of plant biomass allocation. Funct Ecol 21: 713-720.

MEIER F, QUELOZ V, FORSTER B, ODERMATT O, ANGST A ET AL (2016) Waldschutz-Überblick 2015. Birmensdorf: Eidgenöss Forsch.anstalt WSL, Ber 45. 34 p.

MOSER B, TEMPERLI C, SCHNEITER G, WOHLGEMUTH T (2010) Potential shift in tree species composition after interaction of fire and drought in the Central Alps. Eur J For Res 129: 625-633.

MOSER B, METSLAID M, WALTHERT L, WASEM U, WOHLGEMUTH T (2015) Verjüngungspotenzial verschiedener Waldföhren- und Fichtenherkünfte bei variabler Trockenheit. Schweiz Z Forstwes 166: 399-407. doi: 10.3188/szf.2015.0399

MOSER B, WALTHERT L, METSLAID M, WASEM U, WOHLGEMUTH T (2017) Spring water deficit and soil conditions matter more than seed origin and summer drought for the establishment of temperate conifers. Oecologia 183: 519-530.

NICOTRA AB, ATKIN OK, BONSER SP, DAVIDSON AM, FINNEGAN EJ ET AL (2010) Plant phenotypic plasticity in a changing climate. Trends Plant Sci 15: 684-692.

OTT E, LÜSCHER F, FREHNER M, BRANG P (1991) Verjüngungsökologische Besonderheiten im Gebirgsfichtenwald im Vergleich zur Bergwaldstufe. Schweiz Z Forstwes 142: 879-904. doi: $10.5169 /$ seals-702332

PERRET-GENTIL A (2015) Caractérisation et comparaison de la résistance au stress hydrique entre différentes provenances de pin sylvestre et pin noir dans le contexte du changement climatique. Neuchâtel: Université de Neuchâtel, Master thesis. 95 p. 
POORTER H, JAGODZINSKI AM, RUIZ-PEINADO R, KUYAH S, LUO YJ ET AL (2015) How does biomass distribution change with size and differ among species? An analysis for 1200 plant species from five continents. New Phytol 208: 736-749.

RICHTER S, KIPFER T, WOHLGEMUTH T, GUERRERO C, GHAZOUL J ET AL (2012) Phenotypic plasticity facilitates resistance to climate change in a highly variable environment. Oecologia 169: 269-279.

RIGLING A, MOSER B, FEICHTINGER L, GÄRTNER H, GIUGGIOLA A ET AL (2018) 20 Jahre Waldföhrensterben im Wallis: Rückblick und aktuelle Resultate. Schweiz Z Forstwes 169: 242-250. doi: 10.3188/szf.2018.0242

SAVAGE M, BROWN PM, FEDDEMA J (1996) The role of climate in a pine forest regeneration pulse in the southwestern United States. Ecoscience 3: 310-318.

\section{Régénération des pins: plasticité et acclimatation dans un climat plus sec}

L'aggravation des sécheresses estivales pourrait compromettre la régénération naturelle des populations de pins sylvestres à basse altitude dans la vallée du Rhône. Les expérimentations de culture au pied des steppes rocheuses ont montré que la germination et l'établissement des pins sylvestres étaient surtout limités par l'absence de précipitations printanières et, dans une moindre mesure, par la sécheresse estivale ou l'élévation des températures. Les plants de pins sylvestres réagissaient rapidement aux périodes sèches en favorisant la croissance racinaire au détriment des pousses. Dès la deuxième année, les plants étaient déjà armés contre la sécheresse, de sorte que la mortalité due à une sécheresse printanière et estivale étendue, comparable à celles qui ont marquées I'histoire de Sion à douze reprises au cours des 154 dernières années, reste limitée. Ce n'est qu'à la suite d'une sécheresse estivale extrême - pas d'eau de juin à septembre que $14.7 \%$ des plants de pins sylvestres dépérirent. Etonnamment, les individus survivants ont néanmoins réussi à s'acclimater à de telles sécheresses extrêmes. La troisième année, après une nouvelle sécheresse estivale, la mortalité est descendue à moins de 5\%. En général, les plants de pins sylvestres avaient une réaction très plastique, à savoir qu'ils adaptent leur croissance aux conditions environnementales rencontrées. Une adaptation génétique a même été constatée: les pins sylvestres provenant des régions où la sécheresse estivale est prononcée, telles que les régions de basse altitude dans la vallée du Rhône, produisaient plus de biomasse que leurs congénères des régions plus humides, indépendamment du traitement. Les pins noirs réagissaient de façon similaire aux pins sylvestres, mais leur croissance était plus rapide et ils survivaient sensiblement mieux aux sécheresses estivales. Ces résultats montrent que les pins sylvestres à basse altitude de la vallée du Rhône font partie des provenances européennes les plus résistantes à la sécheresse. Leur plasticité et leur capacité d'acclimatation rapide à la sécheresse pourraient, également à l'avenir, être à l'origine d'une régénération naturelle à basse altitude dans la vallée du Rhône, même si moins fréquemment qu'aujourd'hui.
THORNTHWAITE CW (1948) An approach toward a rational classification of climate. Geogr Rev 38: 55-94.

VALLADARES F, SÁNCHEZ-GÓMEZ D, ZAVALA MA (2006) Quantitative estimation of phenotypic plasticity: bridging the gap between the evolutionary concept and its ecological applications. J Ecol 94: 1103-1116.

WOHLGEMUTH T, DOUBLET V, NUSSBAUMER C, FEICHTINGER L, RIGLING A (2018) Baumartenwechsel in den Walliser Waldföhrenwäldern verstärkt nach grossen Störungen. Schweiz Z Forstwes 169: 260-268. doi: 10.3188/szf.2018.0260

ZACKRISSON O, NILSSON MC, STEIJLEN I, HÖRNBERG G (1995) Regeneration pulses and climate-vegetation interactions in nonpyrogenic boreal Scots pine stands. J Ecol 83: 469-483.

\section{Pine regeneration: plasticity and acclimation in a dryer climate}

Increasing summer drought might limit the natural regeneration of Scots pine stands at low elevations of the Rhone valley. Common garden experiments at the forest-steppe ecotone have shown that emergence and establishment of Scots pine primarily depend on spring precipitation and, to a minor degree, on summer drought and rising temperatures. Scots pine seedlings acclimated rapidly to drought periods by favouring root to shoot growth. In the second year, the saplings were already adapted to drought so that most of them survived an extended spring and summer drought, as recorded at Sion twelve times during the last 154 years. Only an extreme summer drought - no water from June to September-killed $14.7 \%$ of the Scots pine saplings. Surprisingly, they were even able to acclimate to such extreme drought events: after the same extreme summer drought in the third year, mortality dropped below 5\%. In general, the Scots pine was very plastic, i.e. seedlings and saplings changed their phenotype depending on environmental conditions. But we also found genetic adaptation: Scots pine originating from regions with pronounced summer drought, including populations from lower elevations in the Rhone valley, produced more biomass than those from moister regions in all treatment combinations. Black pine reacted similarly to the treatments like Scots pine, but it grew faster and more saplings survived the first extreme summer drought. These results show that Scots pine from low elevations of the Rhone valley is one of the most drought-tolerant provenances in Europe. Thanks to its high phenotypic plasticity and the ability of seedlings and saplings to acclimate to drought on a short time scale, natural regeneration of Scot pine at low elevations of the Rhone valley is likely to occur also under future conditions, but maybe less frequent than today. 